\title{
Efficient and accurate whole genome assembly and methylome profiling of $E$. coli
}

\author{
Jason G Powers*, Victor J Weigman, Jenny Shu, John M Pufky, Donald Cox and Patrick Hurban
}

\begin{abstract}
Background: With the price of next generation sequencing steadily decreasing, bacterial genome assembly is now accessible to a wide range of researchers. It is therefore necessary to understand the best methods for generating a genome assembly, specifically, which combination of sequencing and bioinformatics strategies result in the most accurate assemblies. Here, we sequence three E. coli strains on the Illumina MiSeq, Life Technologies Ion Torrent PGM, and Pacific Biosciences RS. We then perform genome assemblies on all three datasets alone or in combination to determine the best methods for the assembly of bacterial genomes.

Results: Three E. coli strains - BL21(DE3), Bal225, and DH5a - were sequenced to a depth of 100x on the MiSeq and Ion Torrent machines and to at least $125 \times$ on the PacBio RS. Four assembly methods were examined and compared. The previously published BL21(DE3) genome [GenBank:AM946981.2], allowed us to evaluate the accuracy of each of the BL21(DE3) assemblies. BL21(DE3) PacBio-only assemblies resulted in a 90\% reduction in contigs versus short read only assemblies, while N50 numbers increased by over 7-fold. Strikingly, the number of SNPs in PacBio-only assemblies were less than half that seen with short read assemblies ( 20 SNPs vs. $~ 50$ SNPs) and indels also saw dramatic reductions ( $\sim 2$ indel $>5$ bp in PacBio-only assemblies vs. $\sim 12$ for short-read only assemblies). Assemblies that used a mixture of PacBio and short read data generally fell in between these two extremes. Use of PacBio sequencing reads also allowed us to call covalent base modifications for the three strains. Each of the strains used here had a known covalent base modification genotype, which was confirmed by PacBio sequencing.

Conclusion: Using data generated solely from the Pacific Biosciences RS, we were able to generate the most complete and accurate de novo assemblies of E. coli strains. We found that the addition of other sequencing technology data offered no improvements over use of PacBio data alone. In addition, the sequencing data from the PacBio RS allowed for sensitive and specific calling of covalent base modifications.
\end{abstract}

Keywords: Genome assembly, Illumina MiSeq, Ion Torrent PGM, PacBio RS, Base modifications, E. coli, Hybrid assembly, $5 \mathrm{mC}$

\section{Background}

Bacteria make up an enormous portion of the world around us. Some estimate that there are $4-6 \times 10^{30}$ prokaryotes on Earth today [1]. They are known to live in virtually every environment on Earth, playing critical roles in both human health (e.g. digestion and disease) and the global ecosystem (e.g. decomposition, oxygen production). Understanding their genetic diversity is a crucial part in understanding how bacteria have evolved to play these various roles. Recent advances in sequencing

\footnotetext{
*Correspondence: jason.powers@quintiles.com

Expression Analysis, A Quintiles Company, Durham NC 27713, USA
}

have made these studies more accessible than ever, with large amounts of sequencing data readily generated from a variety of machines at both a reasonable price and with turnaround times measured in days, not weeks. Increasingly, this accessibility is being applied to both research and clinical studies and is revolutionizing our understanding of bacterial genetics and their diversity. These rapid gains in understanding of bacterial genetics promises to exponentially expand our understanding of the interplay between bacteria, the environment, and human health.

Before such promises can be fulfilled it is important to develop best practices for how the genetic profiles of microbes are studied. Which machines are used to sequence 
the bacteria, the reagents used to prepare the DNA for sequencing, and the software used to analyze the resulting data all influence the depth of knowledge one can gain from any given sequencing experiment. An important step in understanding the genetics of bacteria, or indeed any organism, is to understand the genome - its structure, size and gene organization. Assembling these genomes from next generation sequencing data is thus a critical task towards fully understanding bacterial functions and phylogenetic relationships - an assembly that is highly fractured or incorrect can impede further studies of gene expression, function, and phylogenetic relationships, while accurate assemblies open the door to a wealth of further studies.

Two benchtop sequencers dominate the market today, the Ion Torrent PGM from Life Technologies [2] and the MiSeq from Illumina [3]. Both facilitate rapid, costeffective sequencing. Data generated from these machines can be paired-end or single-end, and can range from $150 \mathrm{nt}$ to $\sim 400 \mathrm{nt}$ in length. Assembling a genome of several million bases or more with reads of this length is a complicated process, although there are a number of freely-available tools to assemble the genome of bacteria using data generated from these machines [4]. Although read lengths continue to improve on these instruments, we will refer to this method of assembly using the historical nomenclature of "short read only assembly" (Figure 1A) [5].

Just as the Ion Torrent and MiSeq can sequence a bacterial genome in less than a day, so can a third sequencing platform, the Pacific Biosciences RS (PacBio). In addition to fast sequencing reactions, the PacBio distinguishes itself from the other two machines in at least three ways. First, the PacBio produces very long reads, some as long as $20 \mathrm{~kb}$, with average read lengths of 4-5 kb, making the single, continuous reads generated by this machine an order of magnitude longer than any other [6]. Second, the library preparation process does not include an amplification step, meaning DNA is sequenced in its native form, a single molecule at a time. This style of sequencing in turn allows for the third distinguishing characteristic of the PacBio - the ability to call covalent base modifications [7]. While understanding the nucleotide sequence of bacterial genomes is an important step in understanding the biology of any given bacterial species, knowledge of the epigenome will also prove to be useful in elucidating biological functions, and indeed has already been applied to pathogenic bacteria [8,9]. Recent work has also defined a roadmap for extending base modification identification to RNA samples, using the HIV-1 reverse transcriptase enzyme instead of a DNA polymerase, providing another sequencing application unique to the PacBio [10,11].
The long read lengths generated by PacBio sequencing come at a price: the average base quality of any given base is substantially lower than that of either the Illumina or Life Technology machines. Still, the long read lengths offer a significant advantage during assembly, and this has led at least two groups to design algorithms that correct the errors found in long PacBio reads using the shorter, yet higher accuracy reads from the Illumina and Life Technology machines [12,13]. Referred to in this paper as Hybrid Assembly, these error corrected reads are more amenable to traditional assembly using overlap graph assemblers like the Celera software package (Figure 1B) [14]. More recently, both PacBio and the research group responsible for Celera have released versions of their software packages which selfcorrects PacBio reads by aligning reads to each other and generating consensus sequences [15-17]. These selfcorrected, PacBio-only reads can be fed into the same assemblers as the hybrid reads (Figure 1C).

Bacterial genome sequencing and assembly is not a new phenomenon, and indeed many researchers have sequenced bacterial genomes and generated draft assemblies [18]. It is therefore not uncommon for researchers to look to improve upon their pre-existing assemblies through a process referred to here as Hybrid Scaffolding (Figure 1D). In this method, a pre-existing bacterial assembly is improved by using PacBio long reads to connect two pre-assembled contigs into a longer sequence called a scaffold. These scaffolds consist of the previously assembled contigs connected by a string of N's in between, called gaps. Many of these intervening gaps can subsequently be filled using PBJelly software, ultimately resulting in a more complete assembly [19].

In this report, we address best practices for bacterial assembly by sequencing the well characterized E. coli strain BL21(DE3) $[20,21]$ on the Illumina MiSeq, Ion Torrent, and PacBio, performing de novo assemblies under a variety of conditions, and comparing those assemblies to the published genome. We examine the four primary methods of bacterial assembly - short read only assemblies, hybrid scaffolding, hybrid assembly, and PacBio-only assembly. We then extend those results to sequence and assemble two other E. coli strains, Bal225 and the common laboratory strain DH5 $\alpha$ [22].

Finally, with assembled bacterial strains in hand, we use PacBio sequencing to examine covalent base modifications present in each of the bacterial strains. The three predominant methyltransferases in E. coli are the DAM methylase, the DCM methylase, and the EcoKI methylase. Each methylase has a specific base substrate within a larger sequence motif which it targets for methylation. The DAM methylase targets the N6 position of the adenine in the sequence motif GA*TC. The DCM methylase specifically methylates the second cytosine in the sequence motifs 

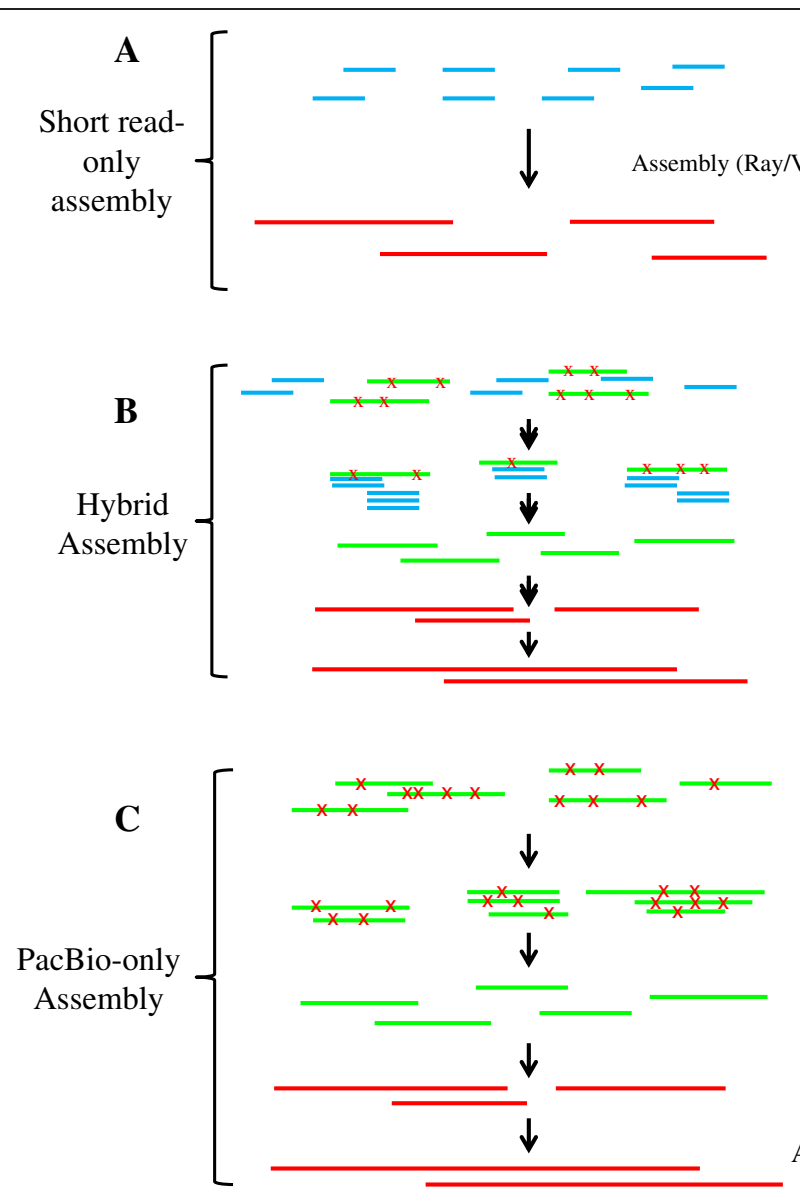

Assembled contigs, scaffolded and filled (see 1D)

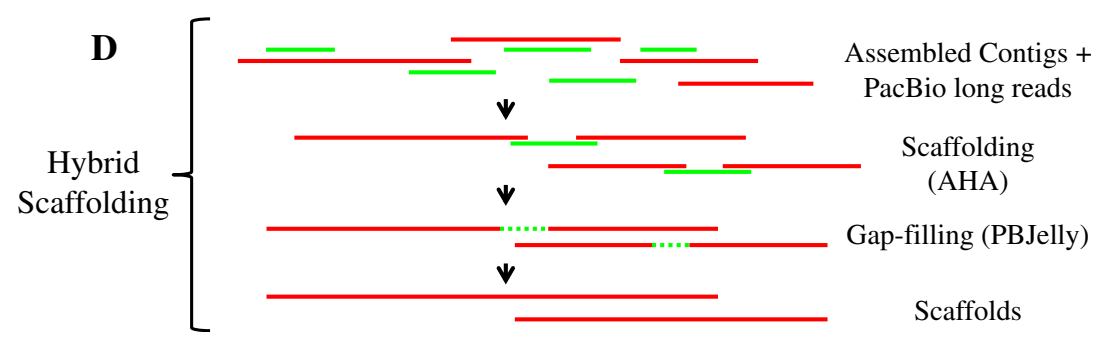

Figure 1 The four bacterial assembly strategies examined here. A. Short read only assembly. Short next generation sequencing reads generated by the Illumina or Life Technologies platforms (in blue) are assembled with any number of assembly software packages into contiguous sequences (contigs; in red). Here, the software packages Velvet, Ray, and MIRA were evaluated. B. Hybrid Assembly. In this method of assembly, short, higher accuracy reads from the Illumina/Life Technologies platforms (blue), error correct the long, lower accuracy PacBio reads (in green with red Xs denoting basecalling errors) via alignment. The software package pacBioToCA was used for this purpose. These error-corrected reads are then assembled into contigs (in red) using a software package suited to long read assembly such as the Celera assembler. Assembled contigs can be further scaffolded and gap-filled as in D. C. In PacBio-only assembly, long reads are aligned to each other, enabling self-correction. These self-corrected reads are assembled into contigs and can be further scaffolded and gap-filled as in $\mathbf{D}$. D. Hybrid scaffolding, in this method, preassembled contigs (red), derived from any of the methods laid out in A-C, are scaffolded with long PacBio reads (in green). These long scaffolds, which can contain long strings of "N"s (dotted green lines) in between the contigs, are then gap-filled with the program PBJelly.

CC*AGG and CC*TGG. Finally, the EcoKI methylase modifies the second or third adenine in the sequence motifs $\mathrm{AA}^{*} \mathrm{C}(\mathrm{N6}) \mathrm{GTGC}$ and $\mathrm{GCA}$ *C(N6)GTT, respectively [23-28]. The three E. coli strains in this study were chosen specifically to address the accuracy and specificity of the PacBio sequence data and associated software to call these modifications on real world data. Whereas DH5 $\alpha$ has all three enzymes, BL21(DE3) is deficient in EcoKI and DCM, while Bal225 lacks the DAM and DCM methylases.

\section{Results}

\section{Short read assembly}

We began our assembly comparisons with BL21(DE3), since the completed genome is available from NCBI, and 
thus provides a reference against which we can compare the various de novo assemblies [GenBank:AM946981.2, 21]. BL21(DE3) was sequenced to a depth of over 100x on both the MiSeq and Ion Torrent PGM machines. MiSeq reads were paired end, 150 bp in length, while the Ion Torrent reads were single-end, approximately $200 \mathrm{bp}$ in length on average (Additional file 1: Table S1).

Several short read assembly software packages are available, and we chose to examine three: Velvet, Ray, and MIRA [29-31]. Ray and Velvet have both been extensively used and validated in competitions such as Assemblathon 1 and 2, while MIRA has been widely used for over a decade [32,33]. To investigate the effects of assembly at lower coverages, we performed assemblies using coverages of $25 \times, 50 \times, 75 \times$, and $100 \times$, by randomly downsampling using the custom script randomFQ [34].

We first assembled data from both benchtop sequencers using Velvet, an extensively used de Bruijn graph assembler [29]. Unfortunately, Velvet's assembly methodology resulted in very poor performance for Ion Torrent assemblies, and we therefore only report the data for MiSeq assemblies. As a de Bruijn graph assembler, Velvet was originally published using Solexa data, and the poor performance with Ion Torrent data is likely due to its inability to cope with the Ion Torrent error profile. For the MiSeq data we assembled the data using
12 different Kmers, ranging from 21 to 63, for each of the different coverage depths, resulting in $48 \mathrm{MiSeq}$ assemblies with Velvet. Each of these assemblies was examined on the basis of contig number, max contig length, percent of contigs $>500$, and 21 bp dup-mer percentage. The $21 \mathrm{bp}$ dup-mer statistic is based on the number of unique $21 \mathrm{bp}$ kmers. The statistic, (\#21 bpkmers occurring $>1$ time)/(total \# of unique $21 \mathrm{bp}$ kmers), can be used to generally evaluate the number of expanded or collapsed repeats, especially when one knows the expected dup-mer number (based on a known reference). A lower than expected number suggests the presence of collapsed repeats, while a higher than expected number indicates expanded repeats. For the reference BL21(DE3) genome, the $21 \mathrm{bp}$ dup-mer is 0.7. Taking all of these statistics under consideration, the most complete Velvet assembly for each of the MiSeq coverages is reported in Table 1.

Next we examined Ray, another de Bruijn graph assembler [30]. Both MiSeq and Ion Torrent data were assembled across a variety of kmer sizes (12 per coverage for a total of 96 different assemblies). Each assembly was evaluated using the same metrics used for Velvet to determine the most complete assembly for each coverage/ data source type. Unlike Velvet, Ray was able to assemble the Ion Torrent data with reasonable results,

Table 1 Short read only assembly statistics

\begin{tabular}{|c|c|c|c|c|c|c|c|c|c|c|}
\hline Assembler & $\begin{array}{l}\text { Data } \\
\text { type }\end{array}$ & kmer & $\begin{array}{l}\text { Approx. } \\
\text { coverage }\end{array}$ & \# Contigs & $\begin{array}{c}\% \text { Contigs }> \\
500\end{array}$ & $\begin{array}{l}\text { Max contig } \\
\text { size }\end{array}$ & N50 & $\begin{array}{c}\text { Dup-mer } \\
21\end{array}$ & $\begin{array}{l}\text { Assembly } \\
\text { size }\end{array}$ & $\begin{array}{c}\% \text { Assembly } \\
\text { size }\end{array}$ \\
\hline \multirow{4}{*}{ Velvet } & \multirow{4}{*}{ MiSeq } & 59 & $100 x$ & 154 & $54.55 \%$ & 430066 & 119241 & 0.28 & 4493252 & $98.56 \%$ \\
\hline & & 59 & $75 x$ & 156 & $53.21 \%$ & 413653 & 119171 & 0.30 & 4493894 & $98.57 \%$ \\
\hline & & 59 & $50 x$ & 144 & $55.56 \%$ & 415116 & 118491 & 0.28 & 4493686 & $98.57 \%$ \\
\hline & & 59 & $25 x$ & 244 & $68.03 \%$ & 212504 & 48994 & 0.26 & 4494206 & $98.58 \%$ \\
\hline \multirow{8}{*}{ Ray } & \multirow{4}{*}{ MiSeq } & 36 & $100 X$ & 93 & $78.49 \%$ & 332975 & 111161 & 1.67 & 4565040 & $100.13 \%$ \\
\hline & & 36 & $75 x$ & 90 & $85.56 \%$ & 350117 & 111227 & 0.47 & 4550209 & $99.81 \%$ \\
\hline & & 36 & $50 x$ & 100 & $90.00 \%$ & 213879 & 86298 & 0.45 & 4495395 & $98.61 \%$ \\
\hline & & 36 & $25 x$ & 292 & $93.15 \%$ & 79672 & 26198 & 0.5 & 4499709 & $98.70 \%$ \\
\hline & \multirow{4}{*}{ Ion } & 29 & $100 x$ & 734 & $88.56 \%$ & 34241 & 10008 & 2.34 & 4545446 & $99.70 \%$ \\
\hline & & 29 & $75 x$ & 578 & $87.02 \%$ & 58878 & 13621 & 1.64 & 4493104 & $98.56 \%$ \\
\hline & & 29 & $50 x$ & 440 & $85.00 \%$ & 85800 & 18468 & 1.01 & 4499305 & $98.69 \%$ \\
\hline & & 29 & $25 X$ & 415 & $86.51 \%$ & 75474 & 19997 & 0.36 & 4470372 & $98.06 \%$ \\
\hline \multirow{8}{*}{ MIRA } & \multirow{4}{*}{ MiSeq } & $\mathrm{n} / \mathrm{a}$ & $100 x$ & 1260 & $6.51 \%$ & 388423 & 115369 & 2.16 & 4754899 & $104.30 \%$ \\
\hline & & $\mathrm{n} / \mathrm{a}$ & $75 X$ & 457 & $22.98 \%$ & 284700 & 96674 & 0.85 & 4630082 & $101.56 \%$ \\
\hline & & $n / a$ & $50 x$ & 321 & $51.09 \%$ & 221854 & 48362 & 1.01 & 4589987 & $100.68 \%$ \\
\hline & & $\mathrm{n} / \mathrm{a}$ & $25 x$ & 1071 & $74.51 \%$ & 33745 & 8703 & 1.31 & 4545144 & $99.70 \%$ \\
\hline & \multirow{4}{*}{ Ion } & $\mathrm{n} / \mathrm{a}$ & $100 x$ & 697 & $10.33 \%$ & 493665 & 180738 & 2.34 & 4763496 & $104.49 \%$ \\
\hline & & $\mathrm{n} / \mathrm{a}$ & $75 x$ & 429 & $19.58 \%$ & 401639 & 128626 & 1.60 & 4674816 & $102.54 \%$ \\
\hline & & $\mathrm{n} / \mathrm{a}$ & $50 x$ & 221 & $37.10 \%$ & 351325 & 144583 & 1.03 & 4598473 & $100.87 \%$ \\
\hline & & $n / a$ & $25 X$ & 153 & $62.75 \%$ & 281400 & 106822 & 0.73 & 4559337 & $100.01 \%$ \\
\hline
\end{tabular}

The bolded assemblies represent the best assembly for the specified combination of sequencer and software. 
however still performed better overall with the MiSeq data, again likely because de Bruijn graph assemblers do poorly with lower quality reads The most complete assemblies for each combination of data type and coverage are reported in Table 1.

Finally, the MIRA assembler was applied to our datasets [31]. MIRA produced the most complete Ion Torrent assembly, with optimal results produced with $25 \times$ coverage. In contrast to Velvet and Ray, the MIRA assembler is an overlap graph based assembler and has a specific parameter set tailored to Ion Torrent data, which may account for these superior results. On the other hand, MIRA struggled with the MiSeq data, possibly because these libraries were constructed using the Illumina Nextera kit. Nextera kits utilize transposonmediated fragmentation, and in our hands the resulting fragments had bimodal insert-size distributions, as opposed to the typical normal distributions that are characteristic of other fragmentation methods, such as acoustic methods (Additional file 2: Figure S1). Again, the most completex assemblies based on contig number, percent of contigs $>500$, max contig size, etc. are reported in Table 1.

After determining the most complete short read assemblies for each combination of assembler/coverage/data type we were left with 20 different assemblies (four Velvet assemblies and eight each for Ray and MIRA). While statistics such as number of contigs, percent of contigs $>500$, and max contig size can be used to evaluate assembly completeness, they are poor measures of assembly correctness. To further evaluate the assemblies based on correctness, each of the twenty most complete assemblies were run through an assembly evaluation script utilizing the MUMmer toolkit against the published BL21(DE3) genome sequence $[35,36]$. This package generates many statistics about the accuracy of an assembly compared to a reference. A selection of these statistics can be seen in Table 2, and based on these statistics coupled with completeness statistics in Table 1, five different assemblies were chosen as the best for each combination of assembler/data type (highlighted in bold in Tables 1 and 2). To better visualize assembly-correctness, dot plots for these five assemblies were generated (Figure 2). These figures, when combined with the statistics in Tables 1 and 2 , demonstrate that the most accurate assemblies were those performed by Ray.

\section{Hybrid scaffolding}

In addition to the short read data, we sequenced BL21 (DE3) to $185 \mathrm{X}$ coverage on the Pacific Biosciences RS. Raw PacBio reads averaged $\sim 3.7 \mathrm{~kb}$ in length. This dropped to approximately $2 \mathrm{~kb}$ in length after adapter removal, which reflects the average library DNA insert size (Additional file 1: Table S1). Each of the five short- read assemblies were improved by connecting the contigs with PacBio long reads. In this two step process, long PacBio reads are first used to connect two distinct contigs. The connected contigs, commonly referred to as scaffolds, are joined with unknown intervening sequences labeled as N's. These strings of N's, called gaps, can then be filled in using the PBJelly software package [19]. Using the AHA scaffolder (part of PacBio's SMRT Analysis software package) [15], contigs were scaffolded together using $\sim 25 \times, 55 \times$, or $110 \times$ PacBio long read coverage, and gap-filled with PBJelly.

In all cases examined, the number of un-connected contigs was reduced by $30 \%$ or more. The max sequence length increased by $40 \%$ or better, and in some cases the max sequence length was over twice that obtained with the short read only assemblies. For example, adding in $55 x$ coverage of PacBio to the MIRA/MiSeq assembly increased the maximum sequence length by over 3 fold, from 221,854 to 716,302 . In two thirds of the cases examined, the resulting scaffolds were less than 100 in number, far better than short read only assemblies alone, for which only 1 in 10 resulted in less than 100 contigs (Table 3 ). These scaffolds were largely contiguous, as PBJelly was generally able to fill in the gaps between scaffolded contigs. With the exception of the Ray-Ion Torrent assembly, the un-filled gaps numbered 23 or fewer, with median lengths of less than $250 \mathrm{bp}$. Improvements were seen when incrementally more PacBio long read coverage was added to the scaffolding process, although there were diminishing returns, especially in contig reduction. At least $70 \%$ of total gains in the reduction of contigs were achieved with just $25 \times$ coverage. Similarly, on average $64 \%$ of the maximum sequence length improvements were seen with $25 \times$ coverage, and $60 \%$ of the N50 gains. These results indicate that at least for these assemblies, $25 \times$ PacBio long read coverage was enough to achieve the majority of the gains available using the hybrid scaffolding approach.

While significant gains were seen in assembly completeness, hybrid scaffolding introduced a significant number of errors into the short read assemblies, especially short indels, a well-known error profile of the PacBio (Table 4). The dot-plots underscored the effect that these new errors had on accuracy, with striking degradation of assembly accuracy in some instances (Figure 3). For example, hybrid scaffolding doubled the number of SNPs and indels greater than $5 \mathrm{bp}$, and tripled the number of relocations in the MIRA-Ion Torrent assembly. Indels less than $5 \mathrm{bp}$ went up dramatically in many of the assemblies, in the case of the Ray-MiSeq assembly going from 1 to nearly 200.

\section{Hybrid assembly}

A third method of assembly involves using the short, higher accuracy reads generated by Illumina or Life Technologies 
Table 2 Evaluating the short-read only assemblies

\begin{tabular}{|c|c|c|c|c|c|c|c|c|c|c|}
\hline Assembler & $\begin{array}{l}\text { Data } \\
\text { type }\end{array}$ & kmer & $\begin{array}{l}\text { Approx. } \\
\text { coverage }\end{array}$ & $\begin{array}{c}\text { Avg } \\
\text { identity }\end{array}$ & $\begin{array}{c}\text { Indels }< \\
5 \text { bp }\end{array}$ & $\begin{array}{c}\text { Indels }>= \\
5 \mathrm{bp}\end{array}$ & Inversions & $\begin{array}{l}\text { Unalignable } \\
\text { contigs }\end{array}$ & Relocations & SNPs \\
\hline \multirow{4}{*}{ Velvet } & \multirow{4}{*}{ MiSeq } & 59 & $100 \mathrm{X}$ & 99.99 & 13 & 38 & 2 & 0 & 16 & 42 \\
\hline & & 59 & $75 X$ & 99.99 & 23 & 31 & 0 & 0 & 12 & 110 \\
\hline & & 59 & $50 X$ & 99.99 & 18 & 36 & 1 & 0 & 17 & 88 \\
\hline & & 59 & $25 X$ & 99.99 & 36 & 129 & 0 & 0 & 8 & 96 \\
\hline \multirow{8}{*}{ Ray } & \multirow{5}{*}{ MiSeq } & 36 & $100 \mathrm{X}$ & 100 & 1 & 6 & 2 & 19 & 2 & 9 \\
\hline & & 36 & $75 X$ & 99.99 & 5 & 10 & 0 & 11 & 2 & 35 \\
\hline & & 36 & $50 X$ & 99.99 & 2 & 3 & 0 & 8 & 2 & 26 \\
\hline & & 36 & $25 x$ & 100 & 5 & 5 & 1 & 4 & 4 & 41 \\
\hline & & 29 & $100 x$ & 99.86 & 5645 & 29 & 0 & 4 & 1 & 67 \\
\hline & \multirow{3}{*}{ Ion } & 29 & $75 X$ & 99.9 & 4209 & 14 & 0 & 4 & 1 & 58 \\
\hline & & 29 & $50 X$ & 99.93 & 2784 & 7 & 0 & 0 & 0 & 58 \\
\hline & & 29 & $25 X$ & 99.95 & 1933 & 3 & 0 & 1 & 1 & 54 \\
\hline \multirow{8}{*}{ MIRA } & \multirow{4}{*}{ MiSeq } & $\mathrm{n} / \mathrm{a}$ & $100 x$ & 99.99 & 8 & 6 & 13 & 2 & 15 & 73 \\
\hline & & n/a & $75 X$ & 99.99 & 20 & 4 & 11 & 0 & 11 & 124 \\
\hline & & $n / a$ & $50 X$ & 99.99 & 31 & 5 & 15 & 0 & 9 & 113 \\
\hline & & $\mathrm{n} / \mathrm{a}$ & $25 X$ & 99.99 & 9 & 7 & 7 & 0 & 7 & 81 \\
\hline & \multirow[t]{4}{*}{ Ion } & $\mathrm{n} / \mathrm{a}$ & $100 x$ & 99.98 & 207 & 6 & 11 & 4 & 11 & 50 \\
\hline & & n/a & $75 X$ & 99.98 & 239 & 8 & 14 & 2 & 9 & 42 \\
\hline & & $\mathrm{n} / \mathrm{a}$ & $50 X$ & 99.98 & 292 & 10 & 9 & 1 & 8 & 58 \\
\hline & & $\mathrm{n} / \mathrm{a}$ & $25 X$ & 99.98 & 524 & 10 & 9 & 0 & 3 & 34 \\
\hline
\end{tabular}

The bolded assemblies represent the best assembly for the specified combination of sequencer and software.

sequencers to error-correct the long PacBio reads and use those corrected reads for assembly (see Figure 1B). Outside of the Pacific Biosciences analysis package there are at least two pieces of software do this, here we chose pacBioToCA, which is part of the Celera assembler package $[12,13]$.

The 185x PacBio coverage and a downsampled subset $(110 \times)$ were error-corrected with either $100 \times$ coverage of Ion Torrent data or $100 \times$ coverage of MiSeq data. Following this error correction, corrected reads were sorted on length and downsampled to two different coverage targets. Previously published work has demonstrated that the best error-corrected read assemblies result from depths of $12-25 \times$ [12]. We therefore downsampled the errorcorrected reads targeting two coverages of $12-15 \times$ and 20-25x. Statistics for both downsampled data sets for both small read technologies can be found in Additional file 3: Table S2. This left us with four data sets each for the Ion Torrent and MiSeq hybrid correction to assembly 1) the full data-set, error-corrected and downsampled to $12-15 \times, 2$ ) the full data-set errorcorrected, and downsampled to $20-25 \times, 3$ ) the subset of data, error-corrected and downsampled to $12-15 \times$, and 4) the subset, error-corrected and downsampled to 20-

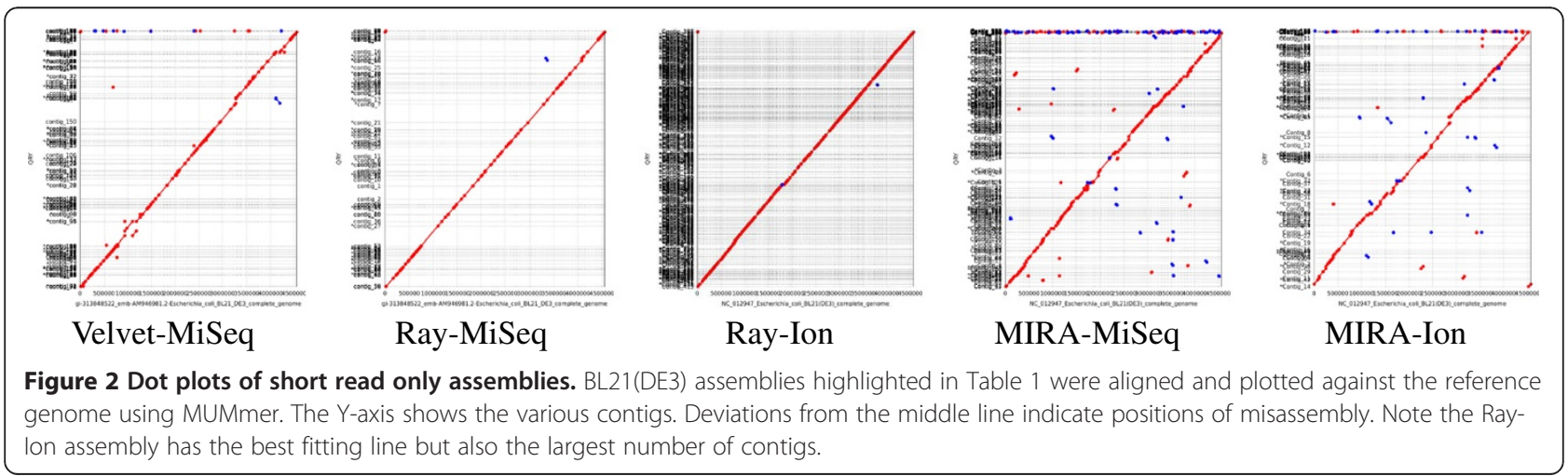


Table 3 Hybrid scaffolding assembly statistics

\begin{tabular}{|c|c|c|c|c|c|c|c|c|c|c|c|c|}
\hline $\begin{array}{l}\text { Original } \\
\text { assembler }\end{array}$ & $\begin{array}{l}\text { Original } \\
\text { data type }\end{array}$ & $\begin{array}{c}\text { Original } \\
\text { coverage }\end{array}$ & $\begin{array}{l}\text { Approx. PacBio } \\
\text { coverage }\end{array}$ & \# Scaffolds & $\%$ improvement & Max scaffold size & $\%$ improvement & N50 & $\%$ improvement & $\begin{array}{l}\text { Total } \\
\text { bases }\end{array}$ & Gaps & $\begin{array}{l}\text { Median } \\
\text { gap size }\end{array}$ \\
\hline \multirow{3}{*}{ Velvet } & \multirow{3}{*}{ MiSeq } & \multirow{3}{*}{$75 x$} & $25 x$ & 94 & $38.96 \%$ & 789973 & $183.69 \%$ & 297566 & $249.55 \%$ & 4530346 & 20 & 141 \\
\hline & & & $55 x$ & 86 & $44.16 \%$ & 1184298 & $275.38 \%$ & 581705 & $487.84 \%$ & 4529781 & 15 & 104 \\
\hline & & & $110 x$ & 77 & $50.00 \%$ & 1107981 & $257.63 \%$ & 581889 & $487.99 \%$ & 4530783 & 14 & 103 \\
\hline \multirow{6}{*}{ Ray } & \multirow{3}{*}{ MiSeq } & \multirow{3}{*}{$50 x$} & $25 x$ & 56 & $39.78 \%$ & 456422 & $137.07 \%$ & 261284 & $235.05 \%$ & 4579304 & 1 & 25 \\
\hline & & & $55 x$ & 47 & $49.46 \%$ & 768167 & $230.70 \%$ & 297488 & $267.62 \%$ & 4574295 & 0 & $\mathrm{n} / \mathrm{a}$ \\
\hline & & & $110 x$ & 52 & $44.09 \%$ & 456422 & $137.07 \%$ & 241297 & $217.07 \%$ & 4578631 & 0 & $\mathrm{n} / \mathrm{a}$ \\
\hline & \multirow{3}{*}{ Ion } & \multirow{3}{*}{$25 x$} & $25 x$ & 92 & $77.83 \%$ & 394228 & $522.34 \%$ & 186135 & $930.81 \%$ & 4605573 & 307 & 201 \\
\hline & & & $55 X$ & 90 & $78.31 \%$ & 324309 & $429.70 \%$ & 179686 & $898.56 \%$ & 4579067 & 62 & 127 \\
\hline & & & $110 x$ & 83 & $80.00 \%$ & 466975 & $618.72 \%$ & 201280 & $1006.55 \%$ & 4592864 & 69 & 175 \\
\hline \multirow{6}{*}{ MIRA } & \multirow{3}{*}{ MiSeq } & \multirow{3}{*}{$50 x$} & $25 X$ & 201 & $37.38 \%$ & 661174 & $298.02 \%$ & 249860 & $516.65 \%$ & 4597589 & 9 & 209 \\
\hline & & & $55 X$ & 179 & $44.24 \%$ & 716302 & $322.87 \%$ & 394266 & $815.24 \%$ & 4600672 & 17 & 110 \\
\hline & & & $110 x$ & 176 & $45.17 \%$ & 759703 & $342.43 \%$ & 433542 & $896.45 \%$ & 4606800 & 23 & 118 \\
\hline & \multirow{3}{*}{ Ion } & \multirow{3}{*}{$25 x$} & $25 x$ & 112 & $26.80 \%$ & 477985 & $169.86 \%$ & 141498 & $132.46 \%$ & 4569060 & 7 & 104 \\
\hline & & & $55 x$ & 105 & $31.37 \%$ & 477631 & $169.73 \%$ & 149865 & $140.29 \%$ & 4569565 & 15 & 120 \\
\hline & & & $110 x$ & 93 & $39.22 \%$ & 537668 & $191.07 \%$ & 200457 & $187.66 \%$ & 4571203 & 17 & 230 \\
\hline
\end{tabular}


Table 4 Evaluation of hybrid scaffolding assemblies

\begin{tabular}{|c|c|c|c|c|c|c|c|c|c|c|}
\hline $\begin{array}{c}\text { Original } \\
\text { assembler }\end{array}$ & $\begin{array}{c}\text { Original } \\
\text { data type }\end{array}$ & $\begin{array}{c}\text { Original } \\
\text { coverage }\end{array}$ & $\begin{array}{c}\text { Approx. PacBio } \\
\text { coverage }\end{array}$ & $\begin{array}{c}\text { Avg } \\
\text { identity }\end{array}$ & Indels $<5$ bp & Indels > = 5 & Inversions & $\begin{array}{c}\text { Missing } \\
\text { assembly contigs }\end{array}$ & Relocation & SNPs \\
\hline \multirow{3}{*}{ Velvet } & \multirow{3}{*}{ MiSeq } & \multirow{3}{*}{$75 x$} & $25 x$ & 99.98 & 416 & 36 & 4 & 0 & 31 & 93 \\
\hline & & & $55 X$ & 99.98 & 471 & 47 & 3 & 0 & 25 & 107 \\
\hline & & & $110 x$ & 99.98 & 299 & 49 & 2 & 0 & 24 & 88 \\
\hline \multirow{6}{*}{ Ray } & \multirow{3}{*}{ MiSeq } & \multirow{3}{*}{$50 x$} & $25 X$ & 99.99 & 191 & 10 & 3 & 19 & 3 & 16 \\
\hline & & & $55 X$ & 99.99 & 210 & 10 & 2 & 19 & 2 & 33 \\
\hline & & & $110 x$ & 99.99 & 185 & 13 & 3 & 19 & 2 & 33 \\
\hline & \multirow{3}{*}{ Ion } & \multirow{3}{*}{$25 x$} & $25 X$ & 99.95 & 2092 & 244 & 8 & 1 & 39 & 65 \\
\hline & & & $55 x$ & 99.93 & 3000 & 93 & 3 & 1 & 13 & 206 \\
\hline & & & $110 x$ & 99.93 & 2732 & 79 & 0 & 1 & 20 & 171 \\
\hline \multirow{6}{*}{ MIRA } & \multirow{3}{*}{ MiSeq } & \multirow{3}{*}{$50 x$} & $25 x$ & 99.98 & 199 & 23 & 18 & 0 & 14 & 138 \\
\hline & & & $55 x$ & 99.98 & 140 & 18 & 19 & 0 & 17 & 103 \\
\hline & & & $110 x$ & 99.98 & 90 & 17 & 21 & 0 & 19 & 92 \\
\hline & \multirow{3}{*}{ Ion } & \multirow{3}{*}{$25 X$} & $25 x$ & 99.97 & 682 & 14 & 12 & 0 & 9 & 59 \\
\hline & & & $55 x$ & 99.97 & 650 & 14 & 12 & 0 & 11 & 92 \\
\hline & & & $110 x$ & 99.97 & 648 & 19 & 13 & 0 & 11 & 74 \\
\hline
\end{tabular}

$25 \times$. The Ion Torrent error-corrected subsets of data were downsampled by selecting for corrected reads greater than 3000 (for 20-25x) and 4000 (for 12-15x) nucleotides in length, while the MiSeq error-corrected subset of data was downsampled for reads greater than 2500 and 3250, respectively. When more PacBio data was added, the downsampling cutoffs were adjusted upwards, to 4000 and 5000 minimum read lengths for the Ion Torrent corrected data and 3250 and 4250 for the MiSeq corrected data.

Each of the eight different corrected data sets was assembled with Celera using 10 different parameters (see Additional file 4: Table S3 for exact parameters), for a total of 80 different assemblies. The most accurate assembly was chosen based on the software package amosvalidate coupled with FRCurve [37,38]. Amosvalidate is a software package designed to identify mis-assemblies based on a variety of metrics, each of which may indicate a poor assembly. Importantly, this software does not rely on a reference, instead attempting to identify assembly problems in de novo assemblies. These potential mis-assembly features can be plotted via FRCurve, with feature counts along the $\mathrm{x}$-axis and total bases covered on the $\mathrm{y}$-axis (see Additional file 5: Figure S2 for a representative curve). The user can then choose the assembly that covers the most bases with the least number of features. Across all samples we chose the assembly with the least features at $90 \%$ coverage of the putative genome size $(4.5 \mathrm{Mb})$. The contigs from the chosen assemblies were then run through the same pipeline as those contigs examined in the Hybrid Scaffolding approach, i.e. scaffolded together with the AHA package and filled in with PBJelly. Assembly statistics can be found in Table 5 .

In terms of assembly accuracy, the MiSeq hybrid assemblies had approximately 10-fold fewer short indels than the Ion Torrent hybrid assemblies. Otherwise, the

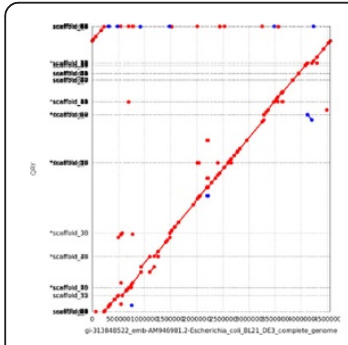

Velvet-MiSeq

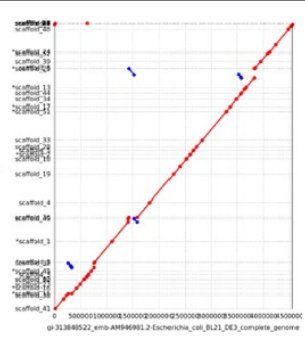

Ray-MiSeq

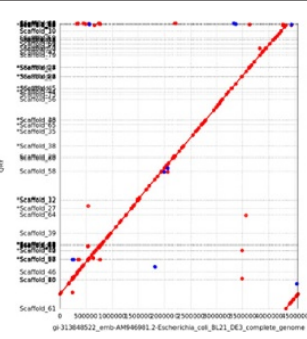

Ray-Ion

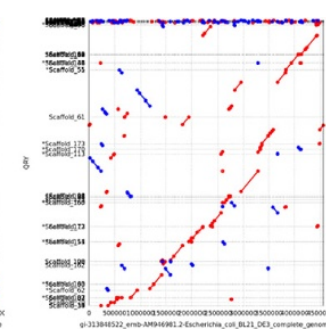

MIRA-MiSeq

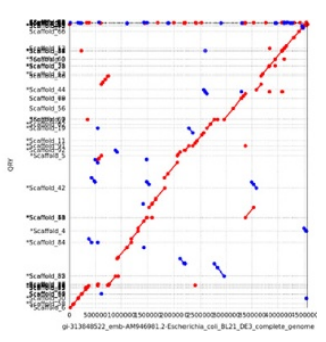

MIRA-Ion

Figure 3 Dot plots of hybrid scaffolding. The assemblies highlighted in Table 1 were scaffolded and gap-filled with varying coverages of PacBio reads. The assembled results with $\sim 100 \times$ PacBio coverage were aligned to the reference BL21(DE3) and dot plots were generated. Note the more contiguous assemblies, but with more errors as compared to those in Figure 2. 
Table 5 Hybrid and PacBio-only assembly statistics

\begin{tabular}{cccccccccccc}
\hline $\begin{array}{c}\text { Correction } \\
\text { method }\end{array}$ & $\begin{array}{c}\text { Starting PacBio } \\
\text { coverage }\end{array}$ & Contigs & N50 & Mean & Max & Total bases & Scaffolds & N50 & Mean & Max & Total bases \\
\hline \multirow{2}{*}{ Ion } & $110 X$ & 25 & 549762 & 188222 & 793657 & 4705573 & 23 & 694467 & 204622 & 1763628 & 4706308 \\
& $185 X$ & 31 & 360777 & 153272 & 869306 & 4751442 & 27 & 1231116 & 176143 & 1631314 & 4755875 \\
\multirow{2}{*}{ MiSeq } & $110 X$ & 42 & 174522 & 107856 & 364773 & 4529989 & 30 & 328144 & 151322 & 588420 & 4539669 \\
& $185 X$ & 49 & 140368 & 92321 & 349122 & 4523744 & 36 & 349122 & 125872 & 648829 & 4531394 \\
\multirow{2}{*}{ Preassembler } & $110 X$ & 22 & 543086 & 212632 & 2026296 & 4677908 & 19 & 696126 & 246383 & 2200813 & 4681278 \\
& $185 X$ & 21 & 737479 & 224642 & 1239560 & 4717483 & 21 & 737479 & 224642 & 1239560 & 4717483 \\
\hline
\end{tabular}

MiSeq and Ion Torrent hybrid assemblies were comparable in terms of accuracy. No assembly produced a contig or scaffold that was un-alignable to the reference genome, and all had identities that were over 99.9\% (Table 6). However, the Ion Torrent hybrid assemblies were more complete than the MiSeq assemblies. When examining the number of contigs, mean, max, and N50 numbers, the Ion Torrent hybrid assemblies produced assemblies that were demonstrably superior to the MiSeq assemblies. For example, with $110 \times$ coverage the Ion Torrent hybrid assembly resulted in a maximum contig length of 793,657, more than twice the maximum seen when the MiSeq was used $(364,773)$. These results can be traced back to the error correction performed with each set of data. In each of the samplings, reads error-corrected with the Ion Torrent ultimately had longer lengths than those error-corrected with the MiSeq data. These longer reads in turn resulted in more complete assemblies. Dot plots were generated with the assembled data showing that both hybrid assemblies were highly accurate and largely complete (Figure 4).

\section{PacBio-only assembly}

Two recent software releases, from PacBio and the Celera Assembler group, allows for the error correction of PacBio reads without the need for short reads from another technology [16,17]. To address the ability of these self-corrected reads to be assembled, we self-corrected both the 110x and 185x BL21(DE3) data sets using the PacBio software package. Prior to error-correction the average base $Q$ score was $\sim 9.6$, post error-correction these qualities increased to between 47 and 57, indicating that error correction was successful. Similar to hybrid assembly, we downsampled based on length to coverages of approximately $12-15 \times$ and 20-25x (Additional file 3: Table S2). Each of the four data sets were assembled with ten different parameter sets using the Celera assembler. We were therefore left with 20 assemblies each for the original 185× PacBio data set and the $110 \times$ data set. The best for each of these coverages was again chosen using amosvalidate, scaffolded with AHA, and gap-filled with PBJelly. Remarkably, self-correction and assembly proved to be more complete than both the Ion Torrent and MiSeq-corrected assemblies across nearly every metric analyzed (Table 5).

The MUMmer/GAGE package showed that the PacBioonly assemblies were not only more complete than the hybrid-assemblies, but largely more accurate. Fewer SNPs, large indels, inversions and relocations were observed in the PacBio-only assembly as compared to the hybrid assemblies (Table 6). This accuracy improved as more PacBio data was added into the assembly, with only 14 SNPs in the final self-assembly, compared to 90 or more in each of the hybrid assemblies. Visual inspection of the dot-plots generated with the hybrid and PacBio-only assemblies also confirmed that PacBio-only assemblies were in fact more accurate than the hybrid-assemblies (Figure 4).

\section{Bal225 and DH5a assemblies}

We next sought to sequence and assemble two additional E. coli strains for which reference genomes were not readily available. Given that the most complete assemblies were achieved with hybrid and PacBio-only assemblies,

Table 6 Evaluating Hybrid and PacBio-only assemblies

\begin{tabular}{|c|c|c|c|c|c|c|c|c|c|}
\hline $\begin{array}{l}\text { Correction } \\
\text { method }\end{array}$ & $\begin{array}{c}\text { Starting PacBio } \\
\text { coverage }\end{array}$ & $\begin{array}{l}\text { \% Assembly } \\
\text { Size }\end{array}$ & $\begin{array}{c}\text { Avg } \\
\text { Identity }\end{array}$ & $\begin{array}{c}\text { Indels }< \\
5 \mathrm{bp}\end{array}$ & $\begin{array}{c}\text { Indels > } \\
=5\end{array}$ & Inversions & $\begin{array}{l}\text { Missing assembly } \\
\text { contigs }\end{array}$ & Relocation & SNPs \\
\hline Ion & $110 x$ & $103.23 \%$ & 99.98 & 859 & 3 & 0 & 0 & 1 & 103 \\
\hline Ion & $185 X$ & $104.32 \%$ & 99.98 & 893 & 4 & 0 & 0 & 1 & 94 \\
\hline MiSeq & $110 x$ & $99.58 \%$ & 99.99 & 149 & 3 & 3 & 0 & 3 & 117 \\
\hline MiSeq & $185 X$ & $99.40 \%$ & 99.99 & 66 & 2 & 1 & 0 & 2 & 104 \\
\hline Self-correction & $110 x$ & $102.68 \%$ & 99.96 & 1489 & 4 & 0 & 0 & 1 & 61 \\
\hline Self-correction & $185 X$ & $103.48 \%$ & 99.99 & 358 & 2 & 0 & 0 & 1 & 14 \\
\hline
\end{tabular}




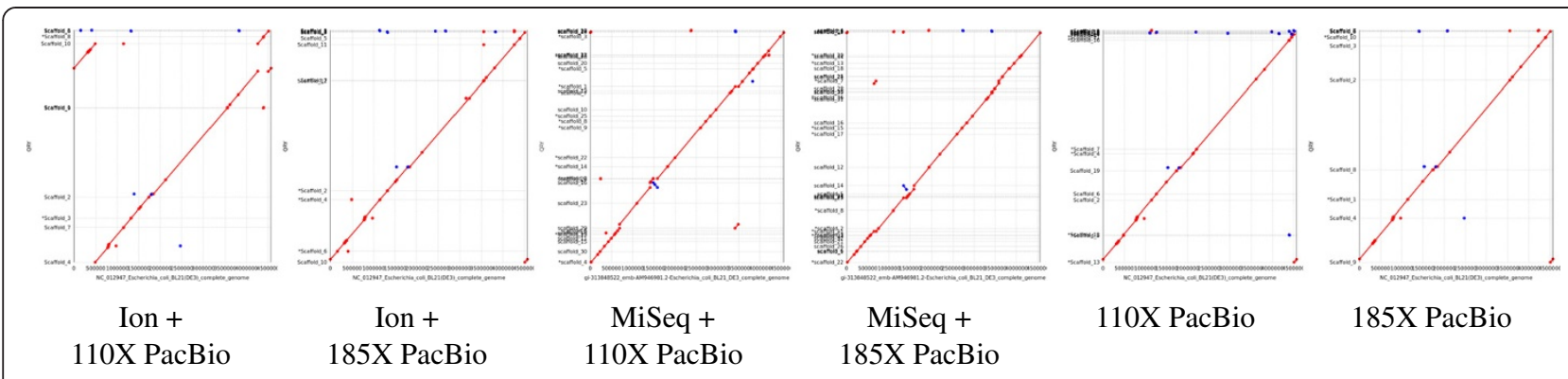

Figure 4 Dot plots of hybrid and PacBio-only assemblies. BL21(DE3) was assembled with varying conditions as described. The best assembly for each combination of technology and PacBio coverage was determined using Amosvalidate. Assemblies were scaffolded with AHA, gap-filled with PBJelly and finally polished with Quiver. The resulting assemblies were aligned to the reference genome and dot plots generated with MUMmer.

short read only assembly and hybrid scaffolding were rejected in favor of these methods. Again, over 100x coverage for each strain was generated on both the MiSeq and the Ion Torrent. PacBio long-insert libraries were prepared for both strains and sequenced to $\sim 135 \times$ coverage for Bal225 (mean read length after adapter removal of $\sim 2 \mathrm{~kb}$ ) and $\sim 200 \times$ coverage for DH5 $\alpha$ (mean read length after adapter removal of $\sim 2.4 \mathrm{~kb}$ ).

As described above for the BL21(DE3) data, the PacBio long reads were corrected with either $100 \times$ MiSeq data or $100 \times$ Ion Torrent data using pacBioToCA, or selfcorrected using PacBio's Preassembler. For Bal225 we corrected all 135× coverage of PacBio long reads. DH $5 \alpha$ data was randomly downsampled to $\sim 100 \times$ coverage and both the full dataset ( $200 \times$ coverage) and this subset were error corrected. For each data set, post-correction downsampling was performed, twenty assemblies for each correction type-coverage combination were performed using the Celera assembler, and the best assemblies chosen.

Final assembly statistics are presented in Table 7 . PacBio-only assemblies of Bal225 were again superior to both types of hybrid-assembly across nearly all metrics analyzed. Less than 20 contigs were assembled, with an N50 of over $1 \mathrm{MB}$, while the Ion Torrent hybrid assembly resulted in 31 contigs (N50 of $0.49 \mathrm{MB}$ ) and the MiSeq hybrid assembly with 54 contigs, and an N50 of only 0.13 MB. Hybrid assembly with Ion Torrent data proved to provide the most complete assembly with the DH5 $\alpha$ strain, although again, the PacBio-only assemblies outperformed the MiSeq-hybrid assemblies by a considerable margin. Specifically, the PacBio-only assembly resulted in less than 20 scaffolds, while the MiSeq assemblies resulted in over 30 scaffolds.

\section{Assembly polishing}

To reduce errors in the BL21(DE3) assembly, we next ran Quiver on each of the hybrid and PacBio-only assemblies [16]. Quiver is a software package that generates high quality consensus sequences by mapping long
PacBio reads against a reference [16]. The assembled scaffolds were used as a reference, and uncorrected PacBio reads were used as the input to generate the consensus. After running Quiver on each assembly, we again evaluated the BL21(DE3) assemblies for correctness using the GAGE and MUMmer package described earlier. We found Quiver to be effective at reducing indels and SNPs, often dramatically improving the accuracy of the assembly (Table 8). In particular, for each of the BL21(DE3) assemblies, SNPs were reduced by $50 \%$ or more. Remarkably, the MiSeq hybrid assembly with 110x PacBio coverage went from 117 SNPs to 2. Small indels were also dramatically reduced, in some cases by more than $80 \%$, demonstrating the utility of running Quiver as a final finishing step.

\section{Base modifications}

A unique feature of data generated with the PacBio is the ability to call base modifications. Identification of these modifications is based on the kinetics of base incorporation. When the interpulse distance ratio of base incorporation differs from expected, it indicates the presence of a modified base [39]. The specific kinetic signatures for $5 \mathrm{mC}, 6 \mathrm{~mA}$, and $4 \mathrm{mC}$ can be reliably modeled and identified from sequencing data. To call $5 \mathrm{mC}$ base modifications, a specialized library preparation is required that increases the intensity signal above background [40]. Current protocols for this library preparation require $500 \mathrm{bp}-1 \mathrm{~kb}$ insert libraries treated with tetracycline. These libraries were generated for all three strains, and sequenced to coverages of at least $60 \times$ for each strain.

The E. coli strains used in this study were chosen specifically for their known methylase genotypes. The DH $5 \alpha$ strain of $E$. coli has functional copies of all three methylases, and therefore all types of methylation should be detectable. As an E. coli strain B bacterium, BL21(DE3) naturally lacks the DCM methylase, and therefore we would expect not to find the methylated sequence motifs CCAGG and CCTGG. BL21(DE3) also lacks the HsdS 
Table 7 Bal225 and DH5a assembly statistics

\begin{tabular}{|c|c|c|c|c|c|c|c|c|c|c|c|c|}
\hline Strain & $\begin{array}{c}\text { Correction } \\
\text { method }\end{array}$ & $\begin{array}{c}\text { Starting PacBio } \\
\text { coverage }\end{array}$ & Contigs & N50 & Mean & Max & $\begin{array}{r}\text { Total } \\
\text { bases }\end{array}$ & Scaffolds & N50 & Mean & Max & $\begin{array}{r}\text { Total } \\
\text { bases }\end{array}$ \\
\hline \multirow{3}{*}{ Bal225 } & Ion & $135 X$ & 31 & 489478 & 155895 & 1066404 & 4832765 & 26 & 2445003 & 185934 & 2445003 & 4834296 \\
\hline & MiSeq & $135 X$ & 54 & 136035 & 84636 & 313301 & 4570378 & 33 & 265913 & 139590 & 436896 & 4606486 \\
\hline & Self-correction & $135 X$ & 19 & 1024938 & 252532 & 2094681 & 4798125 & 16 & 1042373 & 300014 & 2094681 & 4800239 \\
\hline \multirow{6}{*}{ DH5alpha } & Ion & $95 x$ & 17 & 746888 & 276544 & 1350460 & 4701255 & 16 & 1016980 & 293829 & 1350460 & 4701268 \\
\hline & & $198 X$ & 6 & 2698624 & 769397 & 2698624 & 4616384 & 4 & 3138824 & 1154252 & 3138824 & 4617009 \\
\hline & MiSeq & $95 x$ & 49 & 144080 & 92313 & 279069 & 4523338 & 31 & 279069 & 146814 & 664426 & 4551239 \\
\hline & & $198 X$ & 48 & 144514 & 93565 & 356157 & 4491145 & 35 & 189228 & 128837 & 694092 & 4509302 \\
\hline & Self-correction & $95 x$ & 33 & 317772 & 140959 & 711007 & 4651647 & 19 & 503567 & 245691 & 1492059 & 4668131 \\
\hline & & $198 X$ & 35 & 274671 & 133071 & 845967 & 4657500 & 18 & 705151 & 259942 & 971908 & 4678967 \\
\hline
\end{tabular}

subunit of EcoKI, required for sequence recognition, and we therefore do not expect these motifs to be methylated [41]. Finally, Bal225 is known to lack both the DAM and DCM methylases, and therefore we would expect not to find the motifs associated with these methylases to be methylated.

After sequencing the libraries to coverages of $60 \times$ or greater, we used SMRTAnalysis 1.4 to identify base modifications and enriched motifs. This module requires a reference sequence, and for these studies we used the scaffolds that resulted from self-correction and assembly performed earlier. The sequence motifs associated with all three methyltransferases were enriched in the DH5 $\alpha$ samples. Specifically, over $98 \%$ of the motifs associated with the DAM methylase and EcoKI methylases were found to be modified. Detection of $5 \mathrm{mC}$ associated with the DCM methylase was not as strong, but significantly over background, with $40 \%$ of the motifs identified as methylated. As expected, in the Bal225 samples there was no enrichment for the DCM-related sequence motifs or the GATC motif, associated with the DAM methylase. The GATC motif in the BL21(DE3) samples were enriched, with approximately $97 \%$ of the motifs identified as methylated, while lacking all other modifications, as expected. In short, all expected modifications were identified, with no false positives. Table 9 shows the methylation patterns identified for each strain, and Figure 5 shows the location of each motif and each modified motif mapped against the assembled scaffolds.

\section{Discussion}

In this study we explore a variety of methodologies for the de novo assembly of bacterial genomes and analyze the epigenetic base modifications associated with the E.coli strains, BL21(DE3), DH5 $\alpha$, and Bal225. Understanding how best to assemble bacterial genomes de novo is important for at least two reasons. First, bacteria play an important role in nearly all ecological and biological processes on Earth. Full knowledge of how these bacteria interact with the world around them requires an understanding of their underlying genetic architecture. Second, bacterial genomes are relatively simple when compared to more complex eukaryotic genomes. Thus, a firm understanding of how best to assemble bacterial genomes can inform the assembly of larger, more complex genomes.

Here we examined four different methodologies for the assembly of bacterial genomes: short read only assembly, hybrid scaffolding, hybrid assembly, and PacBioonly assembly (Figure 1). To evaluate the effectiveness of

Table 8 Improvements seen with Quiver

\begin{tabular}{|c|c|c|c|c|c|c|c|c|c|c|}
\hline $\begin{array}{l}\text { Correction } \\
\text { method }\end{array}$ & $\begin{array}{c}\text { Starting } \\
\text { PacBio } \\
\text { coverage }\end{array}$ & $\begin{array}{c}\text { Indels } \\
<5 \text { bp before } \\
\text { Quiver }\end{array}$ & $\begin{array}{c}\text { Indels } \\
<5 \text { bp after } \\
\text { Quiver }\end{array}$ & $\begin{array}{c}\text { Indels }<5 \text { bp } \% \\
\text { improvement }\end{array}$ & $\begin{array}{c}\text { Indels }>= \\
5 \text { before } \\
\text { Quiver }\end{array}$ & $\begin{array}{c}\text { Indels }>= \\
5 \text { after } \\
\text { Quiver }\end{array}$ & $\begin{array}{l}\text { Indels }>=5 \% \\
\text { improvement }\end{array}$ & $\begin{array}{l}\text { SNPs } \\
\text { before } \\
\text { Quiver }\end{array}$ & $\begin{array}{l}\text { SNPs } \\
\text { after } \\
\text { Quiver }\end{array}$ & $\begin{array}{c}\text { SNPs \% } \\
\text { improvement }\end{array}$ \\
\hline Ion & $110 x$ & 859 & 292 & $66.01 \%$ & 3 & 2 & $33.33 \%$ & 103 & 30 & $70.87 \%$ \\
\hline Ion & $185 X$ & 893 & 173 & $80.63 \%$ & 4 & 1 & $75.00 \%$ & 94 & 10 & $89.36 \%$ \\
\hline MiSeq & $110 x$ & 149 & 73 & $51.01 \%$ & 3 & 2 & $33.33 \%$ & 117 & 2 & $98.29 \%$ \\
\hline MiSeq & $185 X$ & 66 & 56 & $15.15 \%$ & 2 & 1 & $50.00 \%$ & 104 & 28 & $73.08 \%$ \\
\hline $\begin{array}{c}\text { Self- } \\
\text { correction }\end{array}$ & $110 x$ & 1489 & 237 & $84.08 \%$ & 4 & 2 & $50.00 \%$ & 61 & 36 & $40.98 \%$ \\
\hline $\begin{array}{c}\text { Self- } \\
\text { correction }\end{array}$ & $185 X$ & 358 & 90 & $74.86 \%$ & 2 & 1 & $50.00 \%$ & 14 & 5 & $64.29 \%$ \\
\hline
\end{tabular}


Table 9 Identified methylation patterns

\begin{tabular}{|c|c|c|c|c|c|}
\hline Strain & Genotype & $6 \mathrm{~m}$ & nriched motifs(percent & & $5 \mathrm{mC}$ \\
\hline BL21(DE3) & $\operatorname{dam}(+), \operatorname{dcm}(-), \operatorname{hsdSB}(r B-m B-)$ & GATC (94.83) & & & None \\
\hline Bal225 & $\operatorname{dam}(-), \operatorname{dcm}(-)$ & AAC6NGTGC (97.86) & GCAC6NGTT (96.71) & & None \\
\hline $\mathrm{DH} 5 \mathrm{a}$ & $\operatorname{dam}(+), \operatorname{dcm}(+)$ & AAC6NGTGC (98.15) & GCAC6NGTT (98.82) & GATC (98.49) & CWGG (40.20) \\
\hline
\end{tabular}

The nucleotide bases in bold are the nucleotides that are covalently modified by their respective enzymes.

each strategy we generated $100 \times$ coverage of the three strains on both the Life Technologies Ion Torrent and the Illumina MiSeq and at least $125 \times$ coverage with long-insert reads on the PacBio RS for all three strains.

As was expected, the assemblies with the greatest number of contigs came from assemblies using either the Ion Torrent or MiSeq data alone (short read only). For these studies we examined three commonly used assemblers, Velvet, Ray, and MIRA [29-31]. Both Velvet and Ray are de Bruijn graph-based assemblers. These assemblers are known to be less tolerant of sequencing errors, which may explain why they struggled with the Ion Torrent data whose Q scores were slightly below that of the MiSeq data (28.8 vs. 34.6, Additional file 1: Table S1) [42]. We performed a kmer and coverage parameter sweep with Velvet and the MiSeq data, examining 48 different assemblies. Velvet was capable of assembling the MiSeq data effectively, with generally less than 200 contigs that were typically longer than any of the other short read assemblies. MIRA, which is not a de Bruijn graph assembler, was able to assemble both sets of data, producing the lowest number of contigs with Ion Torrent data, although of the three methods, MIRA had the most trouble with the MiSeq data. Ray stood apart as the most accurate of the three assemblers, based on the number of inversions, relocations, SNPs, and a visual inspection of the associated dot plots (Table 2, Figure 2). These more accurate assemblies did not come at a cost of assembly completeness (Table 1). In particular, the Ray-MiSeq assemblies were often the most complete, with contigs of 100 or less for three of the coverages, the only short read assembler-data combination to achieve such results.

One interesting finding from this study is that more short read coverage does not necessarily guarantee a better assembly. We found that lower coverages, especially for the Ion Torrent data, often resulted in assemblies that were similar to those generated with higher coverage. This is not entirely unexpected for the MIRA assemblies, as overlap graph based assemblers are less tolerant of high coverage [42]. However this observation held true for the Ray-Ion Torrent assemblies as well. We should also note here that while it is typically thought
A

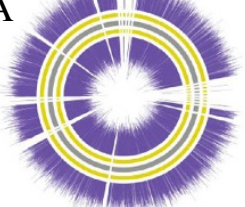

BL21(DE3) - GATC
B

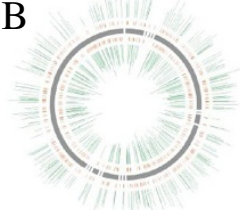

Bal225 -

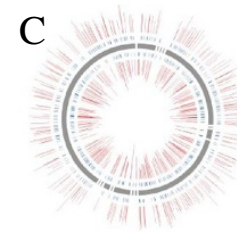

Bal225 -

\section{GCAC6NGTT \\ AAC6NGTGC}

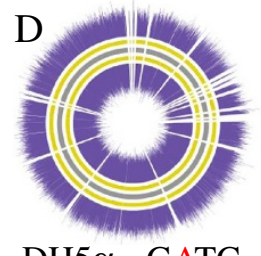

DH5 $\alpha-$ GATC
E

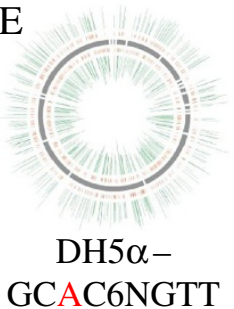

F

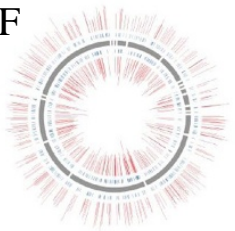

DH5 $\alpha-$

AAC6NGTGC

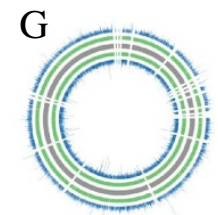

DH5 $\alpha-$ CCWGG

Figure $\mathbf{5}$ Circos plots of base modifications. The PacBio-only assembly was fed into SMRTAnalysis 1.4 and base modifications were called. In each figure, the assembled contigs are plotted as the inner grey bars. On either side of these grey contigs, the short lines indicate motif positions in the genome (the plus sense and minus sense are plotted). Outside of those are the location of the modifications and the intensity of those modifications. A. BL21(DE3) - yellow bars are positions of GATC motifs, purple are those motifs that are modified. B. Bal225, GCAC6NGTT. C. Bal225, AAC6NGTGC. D. DH5alpha, GATC. E. DH5alpha, GCAC6NGTT. F. DH5alpha, AAC6NGTGC. G. DH5alpha, CCWGG. 
that paired-end data is significantly better for assembly than is single-end data, there was little difference in assembly completeness between the best MiSeq assembly (performed with Ray) and the best Ion Torrent assembly (assembled with MIRA).

After generating these short read assemblies, we chose 1 representative assembly from each data:assembler combination (highlighted in Table 1) and attempted to connect the contigs with long, uncorrected PacBio reads. Hybrid scaffolding resulted in significant assembly improvements for all scenarios examined, with an average of $50 \%$ reduction in contig number across all PacBio coverages and an average of 5 -fold improvements in N50 values. Although much of the gains were realized with just $25 \times$ PacBio coverage, improvements did increase incrementally as more PacBio reads were added to the assembly. The assembly that seemed to benefit the most from hybrid scaffolding was the Ray-Ion Torrent assembly (Table 3). This is not terribly surprising, as the Ray-Ion Torrent assemblies were the most accurate, and yet the most fractured, and therefore should be the easiest to connect. When $110 \times$ PacBio coverage was used in hybrid scaffolding, contigs were reduced by $80 \%$ and the N50 length went up by more than 10-fold. What was surprising was the number of errors introduced by using AHA/PBJelly. Far more relocations, inversions, indels, and SNPs are present in these assemblies than in the short read only assemblies (Table 4 and Figure 3). Errors in hybrid scaffolding represent overly aggressive attempts to connect contigs, some of which are connected erroneously. Others have shown it possible to effectively use PacBio data for scaffolding when implemented as a part of the ALL-PATHS LG sequence assembler recipe [43]. Therefore, it should be possible to reduce the aggressiveness of this process in order to eliminate some of these introduced errors, and others may be resolved by running Quiver post-assembly. Reducing the aggressiveness of contig scaffolding will result in less complete assemblies, but the gains made in accuracy may be acceptable in some circumstances. In spite of these potential errors, we employed the hybrid scaffolding technique on all subsequent assemblies. Often, the goal of assemblies is to achieve as complete an assembly as possible. There are always tradeoffs to be made, but in the end we believed that the gains resulting from scaffolding were worth the potential of introduced errors.

While short read only assemblies are still popular because of the relative newness, cost of entry, and throughput concerns associated with long read sequencing technology, the state of the art in genome assembly lies with the long reads generated by the PacBio. We therefore wanted to see how hybrid assembly and PacBioonly assemblies would compare with short read only assemblies and each other. Unexpectedly, the Ion Torrent error-corrected reads assembled far more efficiently for each of the three strains examined across all coverages and parameter sweeps when compared to MiSeq errorcorrected reads. These results can be traced back to longer corrected reads post-Ion Torrent correction. This may be due to the fact that the Ion Torrent reads themselves are longer than the MiSeq reads. These longer reads should be easier to map back to the PacBio reads, increasing error correction efficiency. It's also possible that differences may be due to the manner in which the MiSeq libraries were generated. MiSeq libraries were made using the Nextera kit, which fractures DNA with transposons as opposed to the mechanical shearing used to create the PacBio and Ion Torrent libraries or chemical shearing typical of other Illumina library preparation kits. The insert sizes associated with these libraries were far more varied than what is typically encountered with Illumina libraries, and this may have contributed to the poorer performance of the MiSeq data in both hybrid assembly and the short read only data assembly using MIRA (Additional file 2: Figure S1).

We used Preassembler with the same PacBio data that was used in the previous analyses. Remarkably, the PacBioonly assemblies were superior to the MiSeq-PacBio hybrid assembly across all strains and coverages examined. Furthermore, the completeness of these assemblies were generally comparable to, and often slightly superior to the best Ion Torrent-PacBio hybrid assemblies (Tables 5 and 7). Perhaps even more impressively, the BL21(DE3) PacBioonly assembly was the most accurate of all three types (Table 6). This accuracy improves even further when one finishes the assembly with Quiver (Table 8).

The assembly results here fall largely in line with two recent papers [16,17]. Chin et al. was the first paper to demonstrate the effectiveness of both self-correction using the PacBio software package and Quiver. In Koren, et al. investigators describe using the Celera assembler package to self-correct PacBio long reads, as well as perform hybrid and short read assemblies. Similar to the results shown here, the investigators found that selfcorrection of PacBio long reads lead to as good, or better, assemblies than hybrid-based approaches, and that assembly polishing with the Quiver package led to highly accurate assemblies [17]. This investigation diverges slightly from these two reports in that we unable to close the genome of the three investigated E. coli strains. Closing the genome of microbes is generally thought to be highly correlated to the number and size of the repetitive elements found in the sequenced genomes. Sequence reads must span the repeat regions in order to properly resolve these elements. When these reads are not present, gaps will occur. Two factors are thus important when considering whether or a bacterial genome can be closed - the expected maximum length of repetitive 
elements in the genome of interest, and the length of the sequencing reads. Read lengths of the corrected reads must be longer than the longest repeat, and have sufficient depth as to cover and resolve the repeat regions. For BL21(DE3), post-error correction reads averaged $5000 \mathrm{nt}$ and $5500 \mathrm{nt}$ with $\sim 20 \mathrm{X}$ coverage. This should be close to the necessary lengths needed to resolve these repetitive elements, but were not sufficient in this case.

It should be noted that in the time since this data has been generated, both Illumina and Life Technologies have introduced sequencing kits that produce even longer reads than what was used here - both platforms yield sequence reads that are twice as long as what was used in this study. These reads will undoubtedly improve assemblies with data generated solely by these machines. Additionally, hybrid assemblies should be improved, as longer short read data seems to result in longer errorcorrected reads. Still, given the difficulty these two technologies have with repetitive sequences and read lengths that still fall far short of those produced by PacBio, it is unlikely that these advancements would alter any of the conclusions made here. However, Illumina has recently purchased a technology that rivals the PacBio in read length, known as Moleculo sequencing. This technology stitches together standard Illumina reads into long reads of approximately $10 \mathrm{~kb}$ in length. These reads have the advantage of being both high quality and long, eliminating the need for error correction. Unfortunately since it is based on stitching together short reads, resolution of repetitive regions is likely to remain difficult.

Until Moleculo becomes widely available, and the question of repetitive sequence resolution can be answered, the PacBio should be the platform of choice for any de novo bacterial assembly. In addition to superior assemblies, the PacBio offers a unique capability - the ability to call covalent base modifications. Currently PacBio software can detect three types of base modifications, $6 \mathrm{~mA}, 5 \mathrm{mC}$, and $4 \mathrm{mC}$. The three strains in this study were specifically chosen to test the specificity and sensitivity of the PacBio sequencer and associated software to call modifications. The BL21(DE3) is a type B strain of E.coli, naturally lacking the DCM methylase, and therefore we expected to see no modifications of the CC $\times$ GG motif in this strain and did not. We failed to find enrichments of the motifs associated with EcoKI, but in contrast, high rates of GATC modification were both expected and found. Bal225 was expected to be both DAM and DCM deficient, and while nearly every EcoKI motif was found to be modified, no enrichments for the GATC or $C C \times G G$ motifs were detected. Finally DH $5 \alpha$ served as a positive control, as it is known to be wild-type for all three methyltransferases. Indeed, we found all three motifs to be modified. For this strain, those motifs associated with $6 \mathrm{~mA}$ were highly modified, while the $5 \mathrm{mC}$ motifs $\mathrm{CC} \times \mathrm{GG}$ were detected as modified $\sim 40 \%$ of the time. $5 \mathrm{mC}$ motifs are more difficult for the PacBio software to detect, and a special library preparation is required to call these modifications [40]. We cannot rule out the possibility that this library preparation (which includes the treatment of DNA with tetracycline) was not $100 \%$ effective in marking all modified bases, however, a recent report indicates that not all CC×GG motifs are modified, and this may explain the lower level of methylation found here [44].

\section{Conclusions}

In summary, we compare and contrast competing methods for the assembly of bacterial genomes, demonstrating that PacBio-only assembly is comparable to hybrid assembly and significantly superior to assemblies performed with short read only data. We go on to demonstrate the sensitivity and specificity of calling base modifications using PacBio data.

Moving forward, the results presented here demonstrate that to obtain the most complete and accurate assembly of a bacterial-sized genome, researchers should generate at least $100 \times$ coverage data on the PacBio. This data should then be self-corrected using the PacBio SMRT Analysis software or the Celera error correction module, and assembled using Celera [14-17]. A recent report demonstrates that if enough long read data is obtained, a single contig will be the end result, however if individual contigs remain, researchers can improve the assembly by scaffolding with AHA, and gap-filling with PBJelly $[15-17,19]$. Finally, using Quiver as a final error correction step will improve the accuracy of the assembly even further and should be implemented to ensure the most accurate assembly possible.

\section{Methods}

\section{E.coli strains}

E. coli strains BL21(DE3), Bal225, and DH5 $\alpha^{\text {mim }}$ (Life Technologies, Inc. Grand Island, NY USA) were grown in LB broth to concentrations of approximately $1 \times 10^{9}$ cells $/ \mathrm{ml}$, and genomic DNA was isolated with Qiagen ${ }^{\circ}$ DNeasy $^{\circ}$ Blood and Tissue Kit (Qiagen, Inc. Germantown, MD USA).

\section{Life technologies ion torrent library preparation and sequencing}

Genomic DNA sequencing was conducted using the Life Technologies Ion Torrent Personal Genome Machine ${ }^{\mathrm{nx}}$ $\left(\mathrm{PGM}^{\mathrm{n}}\right)$. Libraries were made using the Ion Plus Fragment Library Kit (Life Technologies Item \# 4471252). Briefly, purified genomic DNA was fragmented to a size range of approximately $200-300 \mathrm{bp}$ using the Covaris ${ }^{\circ}$ E210 instrument (Covaris Inc. Woburn, MA USA). 
Fragmented DNA was repaired and made blunt ended, then purified using Ampure $\mathrm{XP}^{\oplus}$ Beads (Beckman Coulter Inc. Atlanta, GA USA; Item\# A63880). Ion Sequencing adapters and Ion Express ${ }^{\mathrm{Ts}}$ barcodes (Life Technologies Item \# 4474518) were then ligated to the blunt-ended DNA fragments, purified using Ampure $\mathrm{XP}^{\circ}$ Beads, then size-selected to 330 bp using an E-Gel ${ }^{\odot}$ SizeSelect $^{\text {TM }}$ Agarose Gel (Life Technologies Item \# G661002). The size selected product was amplified by PCR, then purified using Ampure $\mathrm{XP}^{\oplus}$ Beads. The resulting DNA library was quantified using the Agilent Bioanalyzer DNA Chip (Agilent Technologies, Inc. Santa Clara, CA USA; Item \# 5067-4626). The libraries were pooled at equimolar concentrations and clonally amplified and enriched onto Ion Spheres using the Ion One Touch ${ }^{\mathrm{Tm}}$ Template Prep System and the Ion Torrent One Touch ${ }^{\mathrm{TM}}$ PGM 200 Kit (Life Technologies Item \# 4478316). Enriched Templated Ion Spheres were deposited onto a semiconductor chip (Ion $318^{\mathrm{TM}}$ Chip, Life Technologies Item \# 4466617) and sequenced using the $\mathrm{PGM}^{\mathrm{TM}}$ Instrument and the Ion $\mathrm{PGM}^{\mathrm{mM}}$ 200 Seq Kit (Life Technologies Item \# 4474004). Ion Torrent Suite software (version 3.2.1) was used to convert raw signal to Base Calls and generate FASTQ files for subsequent analysis.

\section{Illumina MiSeq library preparation and sequencing}

Bacterial genomic DNA was prepared for sequencing on the Illumina MiSeq using the Nextera DNA Sample Prep Kit (Illumina, Inc. San Diego, CA USA; Item \# FC-1211030). Steps were performed as described in the Nextera DNA Sample Preparation Guide (Item \# 15027987 Rev. B October 2012). Briefly, genomic DNA was tagmented (tagged with PCR adapters and fragmented), followed by purification of tagmented DNA and limited-cycle PCR (during which indexes, sequencing adapters, and common adapters are added for subsequent cluster generation and sequencing). PCR library DNA was then purified using Agencourt AMPure XP beads (Beckman Coulter, Item \# A63882), which excluded very short library fragments. DNA libraries were then quantified using the Qubit assay and qualified using the Agilent Technologies High Sensitivity DNA Kit (Item \# 5067-4626). Purified libraries were pooled and sequenced on the MiSeq using a $2 \times 150$ pairedend protocol. Initial basecalls were converted to fastq files using MiSeq CASAVA software suite [45].

Pacific Biosciences RS library preparation and sequencing Three libraries were prepared for each strain: long insert, long insert with Tet1-treatment and $1 \mathrm{~kb}$ insert with Tet1-treatment. Genomic DNA samples were sheared to target insert size $(10 \mathrm{~kb}$ or $1 \mathrm{~kb})$ depending on the chosen sequencing strategy using a Covaris ${ }^{\oplus}$ Adaptive Focused Acoustics instrument, or the g-Tube, also from the Covaris ${ }^{\oplus}$. Fragmented DNA was then purified using AMPure $^{\odot}$ PB magnetic beads and verified on an Agilent Bioanalyzer DNA Chip. Tet1-treatment was carried out on intended fragmented DNA according to guidelines for using the WiseGene $\mathrm{e}^{\mathrm{TM}} 5$-mC Tet1 oxidation kit for SMRT ${ }^{\bullet}$ sequencing on the Pacific Biosciences ${ }^{\bullet}$ RS (WiseGene LLC., Chicago, IL USA; Item \#K004; Pacific Biosciences Inc. Menlo Park, CA USA). Libraries were subsequently prepared following PacBio guidelines. End-repair was performed, followed by ligation of universal hairpin adapters to produce the SMRTbell library. SMRTBell libraries were verified using Life Technologies Qubit ${ }^{\circ}$ 2.0 and the Agilent Bioanalyzer. The PacBio specific sequencing primer was annealed to the SMRTbell library followed by binding of the polymerase to the primerlibrary complex. Libraries were loaded onto the SMRT cells with the assistance of the MagBead stations and sequenced on the PacBio RS system. Long-insert libraries were sequenced with stage-start settings, and used $1 \times 120$ movies, while the short libraries were sequenced with $2 \times 55$ movies. Both libraries were sequenced using the $\mathrm{C} 2$ chemistry and C2-XL enzyme.

\section{Pre-processing}

Ion Torrent data was de-multiplexed using Ion Torrent Suite software (version 3.2.1). MiSeq data was demultiplexed using internally developed software package fastq-multx (available for free download from https://code. google.com/p/ea-utils/) [34]. Unless otherwise noted, all data was clipped for adapters and quality scores with fastqmcf, also internally developed and available for download [46]. PacBio reads were processed with the SMRT Analysis module RS_Filter_and_Control_Pmodules which removes SMRT bell adapters and spike-in sequences.

\section{Short read assemblies}

Using randomFQ BL21(DE3) reads from the MiSeq and Ion Torrent were downsampled to approximate coverages of $25 \times, 50 \times, 75 \times$, and $100 \times$. MiSeq reads were assembled with the Velvet (v. 1.2.08) assembly software package [29] using default parameters, but with varying kmer lengths. Specifically, kmers were varied from 21 to 63. Statistics such as contig number, N50, and $\max$ contig length were generated from each assembly using the script contig-stats (available for download from eautils), and visually inspected. Based on these statistics, a kmer of 59 was chosen as consistently among the best for the four coverages examined. In a similar manner, assemblies with the Ray assembler (v. 2.1.0) [30] were performed with default settings, again with varying kmer lengths (ranging from 21 to 61). Ray was capable of assembling both the Ion Torrent and MiSeq data. Assemblies were again inspected for completeness, and based on these statistics, a kmer of 36 for the MiSeq data and 
29 for the Ion Torrent data consistently resulted in the best assemblies across the four different coverages. Finally, both the Ion Torrent and MiSeq data were assembled using MIRA (v. 3.9.9) [31]. For the Ion Torrent data, we set the technology to "iontor" and used the default parameters for analysis. We chose not to clip the MiSeq data before loading into MIRA, opting instead to allow MIRA's internal clipping algorithm to perform this step by setting CL:pvlc $=$ on:qc $=$ on and the technology to "solexa".

\section{Hybrid-error correction}

PacBio long reads were error-corrected by 100x coverage of either MiSeq reads or Ion Torrent reads essentially as described [12]. Briefly, MiSeq and Ion Torrent fastq files were converted to pacBioToCA compatible frg files with fastqToCA, also part of the Celera assembler package [14]. These frg files were then used as input, along with the uncorrected reads into pacBioToCA. Post-error correction reads were downsampled based on length to two coverages, between 12 and $25 \times$ using internally developed scripts [34].

\section{Preassembler}

For each strain the associated SMRT cells were loaded into the SMRT Analysis package [15]. SMRT cells were chosen in the interface for use in each set of corrections. Minimum Seed Read Length was chosen to get two coverages between 12 and 25x. The BLASR "-maxLCPLength" option was set to 14, and "Trim FASTQ Output" was turned off [47]. Post-correction we ran trimFastqByQVWindow.py on the corrected fastqs with cutoffs of 19 or 49 , ultimately choosing the cutoff that placed the data in the desired coverage range.

\section{Celera assembly}

Celera assembler (v. 7.0) [14] was used to assemble the corrected reads. Ten different parameter settings for each data set was used, mostly variations of ErrorRates and merSize (Additional file 4: Table S3).

\section{Assembly assessment}

BL21(DE3) assemblies were compared to the NCBI reference using the GAGE script which interfaces with MUMmer [35,36]. Celera assemblies were assessed with amosvalidate and FRCurve as described [37,38].

\section{Scaffolding}

After assembly, contigs were imported into the SMRT Analysis package and used as the reference for A Hybrid Assembler (AHA). PacBio reads were used to scaffold the contigs using default parameters. The resultant scaffolds were gap-filled with PBJelly [19], again using default parameters.

\section{Covalent base modifications}

Base modifications were found using the SMRT Analysis package and the accompanying package RS Modification and Motif Analysis. Circos plots were generated with internally developed scripts and the Circos graphics package [48].

\section{Availability of supporting data}

The data sets supporting the results of this article are available in the SRA repository, (Bal225: PRJNA203022; BL21 (DE3): PRJNA203015). Custom scripts used to analyze the data are available on the ea-utils FASTQ processing utilities website https://code.google.com/p/ea-utils/.

\section{Additional files}

Additional file 1: Table S1. BL21(DE3) sequencing statistics.

Additional file 2; Figure S1. Insert size distribution of MiSeq reads. BL21(DE3) MiSeq reads were aligned to the BL21(DE3) reference and the calculated insert sizes were plotted using $R$ and ggplot2 (in navy blue). Previous data generated with an Illumina TruSeq kit and the E. coli strain $\mathrm{DH} 10 \mathrm{~b}$ was similarly mapped to its reference and insert sizes plotted (in red). Note the bimodal distribution of the BL21(DE3) reads.

Additional file 3: Table S2. Post-error correction statistics.

Additional file 4: Table S3. Celera spec file parameters.

Additional file 5: Figure S2. An example FRCurve. This FRCurve was generated from the BL21(DE3) Ion Torrent hybrid assembly with 185x PacBio coverage and the Celera assembler. Amosvalidate and FRCurve were used to analyze 20 different assemblies. Amosvalidate-identified features (representing potential mis-assemblies) were plotted on the $x$-axis with approximate genome coverage on the $y$-axis. The assembly with the lowest number of features at $95 \%$ genome coverage was identified as the best assembly. Here, that assembly is highlighted in black, and corresponds to Celera spec file parameters "Run 1" in Additional file 4: Table S3.

\section{Abbreviations}

PacBio: Pacific Biosciences; RS: AHA, A Hybrid Scaffolder; DAM: DNA adenine methylase; DCM: DNA cytosine methylase.

\section{Competing interests}

The authors declare that they have no competing interests.

\section{Authors' contributions}

JGP designed the experiments, carried out bioinformatics analysis, and drafted the manuscript. VJW helped design the experiments and draft the manuscript. JS carried out all PacBio molecular biology and sequencing. JMP prepared the Illumina MiSeq libraries. DC prepared Ion Torrent library preparation and sequencing. PH aided in the design and implementation of the experiments. All authors read and approved the final manuscript.

\section{Acknowledgements}

We acknowledge the laboratory of Steven A. Lommel at N.C. State University for providing E. coli genomic DNA for analysis, Matt Nash for assisting in software installation and maintenance during the bioinformatics analysis, and Maria Curtis for running the Illumina MiSeq libraries.

Received: 21 May 2013 Accepted: 26 September 2013

Published: 3 October 2013

\section{References}

1. Whitman WB, Coleman DC, Wiebe WJ: Prokaryotes: the unseen majority. Proc Natl Acad Sci U S A 1998, 95(12):6578-6583.

2. Rothberg JM, Hinz W, Rearick TM, Schultz J, Mileski W, Davey M, Leamon JH, Johnson K, Milgrew MJ, Edwards M, Hoon J, Simons JF, Marran D, Myers JW, 
Davidson JF, Branting A, Nobile JR, Puc BP, Light D, Clark TA, Huber M, Branciforte JT, Stoner IB, Cawley SE, Lyons M, Fu Y, Homer N, Sedova M, Miao X, Reed B, et al: An integrated semiconductor device enabling non-optical genome sequencing. Nature 2011, 475(7356):348-352.

3. Bentley DR, Balasubramanian S, Swerdlow HP, Smith GP, Milton J, Brown CG, Hall KP, Evers DJ, Barnes CL, Bignell HR, Boutell JM, Bryant J, Carter RJ, Keira Cheetham R, Cox AJ, Ellis DJ, Flatbush MR, Gormley NA, Humphray SJ, Irving LJ, Karbelashvili MS, Kirk SM, Li H, Liu X, Maisinger KS, Murray LJ, Obradovic B, Ost T, Parkinson ML, Pratt MR, et al: Accurate whole human genome sequencing using reversible terminator chemistry. Nature 2008, 456(7218):53-59.

4. Zhang W, Chen J, Yang Y, Tang Y, Shang J, Shen B: A practical comparison of De novo genome assembly software tools for next-generation sequencing technologies. PLOS One 2011, 6(3). e17915.

5. Paszkiewicz K, Studholme DJ: De novo assembly of short sequence reads. Brief Bioinform 2010, 11(5):457-472.

6. Eid J, Fehr A, Gray J, Luong K, Lyle J, Otto G, Peluso P, Rank D, Baybayan P, Bettman B, Bibillo A, Bjornson K, Chaudhuri B, Christians F, Cicero R, Clark S, Dalal R, Dewinter A, Dixon J, Foquet M, Gaertner A, Hardenbol P, Heiner C, Hester K, Holden D, Kearns G, Kong X, Kuse R, Lacroix Y, Lin S, et al: Real-time DNA sequencing from single polymerase molecules. Science 2009, 323(5910):133-138.

7. Flusberg BA, Webster DR, Lee JH, Travers KJ, Olivares EC, Clark TA, Korlach J, Turner SW: Direct detection of DNA methylation during single-molecule, real-time sequencing. Nat Methods 2010, 7(6):461-465.

8. Fang G, Munera D, Friedman DI, Mandlik A, Chao MC, Banerjee O, Feng Z, Losic B, Mahajan MC, Jabado OJ, Deikus G, Clark TA, Luong K, Murray IA, Davis BM, Keren-Paz A, Chess A, Roberts RJ, Korlach J, Turner SW, Kumar V, Waldor MK, Schadt EE: Genome-wide mapping of methylated adenine residues in pathogenic Escherichia coli using single-molecule real-time sequencing. Nat Biotechnol 2012, 30(12):1232-1239.

9. Murray IA, Clark TA, Morgan RD, Boitano M, Anton BP, Luong K, Fomenkov A, Turner SW, Korlach J, Roberts RJ: The methylomes of six bacteria. Nucleic Acids Res 2012, 40(22):11450-11462.

10. Saletore Y, Meyer K, Korlach J, Vilfan ID, Jaffrey S, Mason CE: The birth of the epitranscriptome: deciphering the function of RNA modifications. Genome Biol 2012, 13(10):175.

11. Vilfan ID, Tsai YC, Clark TA, Wegener J, Dai Q, Yi C, Pan T, Turner SW, Korlach J: Analysis of RNA base modification and structural rearrangement by single-molecule real-time detection of reverse transcription. J Nanobiotechnol 2013, 11(8).

12. Koren S, Schatz MC, Walenz BP, Martin J, Howard JT, Ganapathy G, Wang Z Rasko DA, McCombie WR, Jarvis ED, Phillippy AM: Hybrid error correction and de novo assembly of single-molecule sequencing reads. Nat Biotechnol 2012, 30(7):693-700.

13. Au KF, Underwood JG, Lee L, Wong WH: Improving PacBio long read accuracy by short read alignment. PLOS One 2012, 7(10). e46679.

14. Miller JR, Delcher AL, Koren S, Venter E, Walenz BP, Brownley A, Johnson J, Li K, Mobarry C, Sutton G: Aggressive assembly of pyrosequencing reads with mates. Bioinformatics 2008, 24(24):2818-2824.

15. Pacific Biosciences, Inc: DevNet: $S M R T^{\circledast}$ Analysis. http://pacificbiosciences. github.io/DevNet/code.html.

16. Chin CS, Alexander DH, Marks P, Klammer AA, Drake J, Heiner C, Clum A, Copeland A, Huddleston J, Eichler EE, Turner SW, Korlach J: Nonhybrid, finished microbial genome assemblies from long-read SMRT sequencing data. Nat Methods 2013, 10:563-569.

17. Koren S, Harhay GP, Smith TP, Bono JL, Harhay DM, McVey SD, Radune D, Bergman NH, Phillippy AM: Reducing assembly complexity of microbial genomes with single-molecule sequencing. Genome Biol 2013, 14(9). R101.

18. Mavromatis K, Land ML, Brettin TS, Quest DJ, Copeland A, Clum A, Goodwin L, Woyke T, Lapidus A, Klenk HP, Cottingham RW, Kyrpides NC: The fast changing landscape of sequencing technologies and their impact on microbial genome assemblies and annotations. PLOS One 2012, 7(12). e48837.

19. English AC, Richards S, Han Y, Wang M, Vee V, Qu J, Qin X, Muzny DM, Reid JG, Worley KC, Gibbs RA: Mind the Gap: upgrading genomes with pacific biosciences RS long-read sequencing technology. PLOS One 2012, 7(11). e47768.

20. Daegelen P, Studier FW, Lenski RE, Cure S, Kim JF: Tracing ancestors and relatives of Escherichia coli $B$, and the derivation of $B$ strains REL606 and BL21(DE3). J Mol Biol 2009, 394(3):634-643.
21. Jeong $H$, Barbe $V$, Lee $C H$, Vallenet $D$, Yu DS, Choi SH, Couloux A, Lee SW, Yoon SH, Cattolico L, Hur CG, Park HS, Ségurens B, Kim SC, Oh TK, Lenski RE, Studier FW, Daegelen P, Kim JF: Genome sequences of Escherichia coli B strains REL606 and BL21(DE3). J Mol Biol 2009, 394(4):644-652.

22. Hanahan D: Studies on transformation of Escherichia coli with plasmids. J Mol Biol 1983, 166:557-580.

23. Marinus MG, Morris NR: Isolation of deoxyribonucleic acid methylase mutants of Escherichia coli K-12. J Bacteriol 1973, 114(3):1143-1150.

24. Bigger $\mathrm{CH}$, Murray $\mathrm{K}$, Murray NE: Recognition sequence of a restriction enzyme. Nat New Biol 1973, 244(131):7-10.

25. Geier GE, Modrich P: Recognition sequence of the dam methylase of Escherichia coli K12 and mode of cleavage of Dpn I endonuclease. J Biol Chem 1979, 254(4):1408-1413.

26. Boyer HW, Chow LT, Dugaiczyk A, Hedgpeth J, Goodman HM: DNA substrate site for the EcoRII restriction endonuclease and modification methylase. Nat New Biol 1973, 244(132):40-43.

27. Kan NC, Lautenberger JA, Edgell MH, Hutchison CA 3rd: The nucleotide sequence recognized by the Escherichia coli $\mathrm{K} 12$ restriction and modification enzymes. J Mol Biol 1979, 130(2):191-209.

28. Kennaway CK, Taylor JE, Song CF, Potrzebowski W, Nicholson W, White JH, Swiderska A, Obarska-Kosinska A, Callow P, Cooper LP, Roberts GA, Artero JB, Bujnicki JM, Trinick J, Kneale GG, Dryden DT: Structure and operation of the DNA-translocating type I DNA restriction enzymes. Genes Dev 2012, 26(1):92-104.

29. Zerbino DR, Birney E: Velvet: algorithms for de novo short read assembly using de Bruijn graphs. Genome Res 2008, 18(5):821-829.

30. Boisvert S, Laviolette F, Corbeil J: Ray: simultaneous assembly of reads from a mix of high-throughput sequencing technologies. $J$ Comput Biol 2010, 17(11):1519-1533.

31. Chevreux B, Wetter $T$, Suhai $S$ : Genome sequence assembly using trace signals and additional sequence information. Hannover, Germany: Computer Science and Biology: Proceedings of the German Conference on Bioinformatics; 1999:45-56.

32. Earl D, Bradnam K, St John J, Darling A, Lin D, Fass J, Yu HO, Buffalo V, Zerbino DR, Diekhans M, Nguyen N, Ariyaratne PN, Sung WK, Ning Z, Haimel M, Simpson JT, Fonseca NA, Birol I, Docking TR, Ho IY, Rokhsar DS, Chikhi R, Lavenier D, Chapuis G, Naquin D, Maillet N, Schatz MC, Kelley DR, Phillippy AM, Koren S, et al: Assemblathon 1: a competitive assessment of de novo short read assembly methods. Genome Res 2011, 21:2224-2241

33. Bradnam KR, Fass JN, Alexandrov A, Baranay P, Bechner M, Birol I, Boisvert S, Chapman JA, Chapuis G, Chikhi R, Chitsaz H, Chou WC, Corbeil J, Del Fabbro C, Docking TR, Durbin R, Earl D, Emrich S, Fedotov P, Fonseca NA, Ganapathy G, Gibbs RA, Gnerre S, Godzaridis E, Goldstein S, Haimel M, Hall G, Haussler D, Hiatt JB, Ho IY, et al: Assemblathon 2: evaluating de novo methods of genome assembly in three vertebrate species. Giga Sci 2013. In press.

34. Aronesty E, ea-utils: Command-line tools for processing biological sequencing data. http://code.google.com/p/ea-utils.

35. Salzberg SL, Phillippy AM, Zimin A, Puiu D, Magoc T, Koren S, Treangen TJ, Schatz MC, Delcher AL, Roberts M, Marçais G, Pop M, Yorke JA: GAGE: a critical evaluation of genome assemblies and assembly algorithms. Genome Res 2012, 22(3):557-567.

36. Kurtz S, Phillippy A, Delcher AL, Smoot M, Shumway M, Antonescu C, Salzberg SL: Versatile and open software for comparing large genomes. Genome Biol 2004, 5(2):R12.

37. Phillippy AM, Schatz MC, Pop M: Genome assembly forensics: finding the elusive mis-assembly. Genome Biol 2008, 9(3):R55.

38. Narzisi G, Mishra B: Comparing De novo genome assembly: the long and short of it. PLOS One 2011, 6(4):e19175.

39. Schadt EE, Banerjee O, Fang G, Feng Z, Wong WH, Zhang X, Kislyuk A, Clark TA, Luong K, Keren-Paz A, Chess A, Kumar V, Chen-Plotkin A, Sondheimer N, Korlach J, Kasarskis A: Modeling kinetic rate variation in third generation DNA sequencing data to detect putative modifications to DNA bases. Genome Res 2012, 23(1):129-141.

40. Clark TA, Lu X, Luong K, Dai Q, Boitano M, Turner SW, He C, Korlach J: Enhanced 5-methylcytosine detection in single-molecule, real-time sequencing via Tet1 oxidation. BMC Biol 2013, 11:4.

41. Wion D, Casadesus J: N6-methyl-adenine: an epigenetic signal for DNA-protein interactions. Nat Rev Microbiol 2006, 4(3):183-192. 
42. Nagarajan N, Pop M: Sequence assembly demystified. Nat Rev 2013, 14:157-167.

43. Ribeiro FJ, Przybylski D, Yin S, Sharpe T, Gnerre S, Abouelleil A, Berlin AM, Montmayeur A, Shea TP, Walker BJ, Young SK, Russ C, Nusbaum C, MacCallum I, Jaffe DB: Finished bacterial genomes from shotgun sequence data. Genome Res 2012, 22(11):2270-2277.

44. Kahramanoglou C, Prieto Al, Khedkar S, Haase B, Gupta A, Benes V, Fraser GM, Luscombe NM, Seshasayee A: Genomics of DNA cytosine methylation in Escherichia coli reveals its role in stationary phase transcription

Nat Commun 2012, 3:886

45. Illumina, Inc: CASAVA. http://support.illumina.com/sequencing/sequencing_ software/casava.ilmn.

46. Aronesty E: Comparison of sequencing utility programs. Open Bioinform $J$ 2013, 7:1-8.

47. Chaisson MJ, Tesler G: Mapping single molecule sequencing reads using basic local alignment with successive refinement (BLASR): theory and application. BMC Bioinforma 2012, 13:238.

48. Krzywinski M, Schein J, Birol I, Connors J, Gascoyne R, Horsman D, Jones SJ, Marra MA: Circos: an information aesthetic for comparative genomics. Genome Res 2009, 19:1639-1645.

doi:10.1186/1471-2164-14-675

Cite this article as: Powers et al.: Efficient and accurate whole genome assembly and methylome profiling of E. coli. BMC Genomics 2013 14:675.

\section{Submit your next manuscript to BioMed Central and take full advantage of:}

- Convenient online submission

- Thorough peer review

- No space constraints or color figure charges

- Immediate publication on acceptance

- Inclusion in PubMed, CAS, Scopus and Google Scholar

- Research which is freely available for redistribution 